\title{
AVALIAÇÃO DA QUALIDADE DE MÉIS DE ABELHAS AFRICANIZADAS E SEM FERRÃO NATIVAS DO NORDESTE DO ESTADO DO PARÁ
}

\author{
EVALUATION OF QUALITY OF HONEY FROM AFRICANIZED AND \\ STINGLESS BEES NATIVES OF THE NORTHEAST OF THE STATE OF \\ PARÁ.
}

\author{
Blenda do Amor Divino Menezes ${ }^{1 *}$ \\ Rafaella de Andrade Mattietto ${ }^{2}$ \\ Lúcia de Fátima Henriques Lourenço ${ }^{1}$ \\ ${ }^{1}$ Universidade Federal do Pará, Belém, PA, Brasil. \\ ${ }^{2}$ EMBRAPA Amazônia Oriental, Belém, PA, Brasil. \\ *Autora para correspondência - blendadivino@gmail.com
}

\section{Resumo}

O objetivo do trabalho foi avaliar a qualidade microbiológica e físico-química do mel in natura (np) e pasteurizado (p) de abelhas africanizadas (Apis mellifera) e de duas espécies de abelhas sem ferrão (Melipona fasciculata e Melipona flavolineata) nativas da região Nordeste do Estado do Pará. As análises físico-químicas foram realizadas de acordo com o recomendado pela legislação brasileira para Apis mellifera e as análises microbiológicas seguiram os métodos oficiais da American Public Health Association. Os méis de Apis mellifera apresentaram-se dentro dos padrões físico-químicos estabelecidos, exceto para os parâmetros açúcares redutores (np: $57,43 \% \pm 1,28$ e p: $58,53 \% \pm 1,91$ ), acidez (np: 62,25 cmol/Kg $\pm 4,15$ e p: $62,67 \mathrm{cmol} / \mathrm{Kg} \pm 5,75$ ) e umidade (np: $21,02 \% \pm 0,17$ e p: $20,12 \% \pm 0,07)$. Os méis de meliponíneos apresentaram-se dentro da faixa observada na literatura para esse tipo de mel. Em quase todas as análises físico-químicas realizadas, os méis estudados mostraramse diferentes entre si, sendo que os méis de meliponíneos apresentam umidade e atividade de água mais elevada, o que os torna mais suscetíveis a deteriorações. Foi observada diferença significativa entre os méis in natura de $A$. mellifera e de meliponíneos quando comparado com seus respectivos méis pasteurizados para as análises de umidade (A. melifera, np: $21,02 \% \pm 0,17$ e p: $20,12 \% \pm 0,07 ; M$. fasciculata, np: $24,33 \% \pm 0,03$ e p: $23,68 \% \pm 0,16$; $M$. flavolineata, np: $28,53 \% \pm 0,02$ e p: $27,40 \% \pm 0,04$ ), sacarose aparente (A. melifera, np: $5,75 \% \pm 0,18$ e p: $3,19 \% \pm 0,23 ;$ M. fasciculata, np: $3,89 \% \pm 0,04$ e p: $1,59 \% \pm 0,03 ;$ M. flavolineata, np: $2,12 \% \pm 0,22$ e p: $1,62 \% \pm 0,02)$ e HMF (A. melifera, np: $20,99 \mathrm{mg} / \mathrm{Kg} \pm 0,13$ e p: $64,36 \mathrm{mg} / \mathrm{Kg} \pm 0,07 ;$ M. fasciculata, np: $6,54 \mathrm{mg} / \mathrm{Kg} \pm 0,14$ e p: 9,46mg/Kg $\pm 0,09 ;$ M. flavolineata, $\mathrm{np}: 3,59 \mathrm{mg} / \mathrm{Kg} \pm 0,14$ e p: $43,19 \mathrm{mg} / \mathrm{Kg} \pm 0,14)$. Todas as amostras submetidas ao processo de pasteurização encontravam-se dentro dos limites microbiológicos estabelecidos pela legislação vigente.

Palavras chaves: Apis melífera; caracterização; mel; meliponíneos; pasteurização 


\begin{abstract}
The objective of this study was to evaluate the microbiological and physicochemical quality of fresh (np) and pasteurized (p) honey from Africanized bees (Apis mellifera) and two species of stingless bees (Melipona fasciculata and Melipona flavolineata), native to the northeastern region of the State of Pará. Physicochemical analyzes were performed according to the Brazilian legislation recommended for Apis mellifera and the microbiological analysis followed the official methods by the American Public Health Association. The honey from Apis mellifera were within the established physicochemical standards, except for the parameters reducing sugars (np: $57.43 \% \pm 1.28$ and p: $58.53 \% \pm 1.91$ ), acidity (np: $62.25 \mathrm{cmol} / \mathrm{Kg} \pm 4.15$ and $\mathrm{p}: 62.67 \mathrm{cmol} / \mathrm{Kg} \pm 5.75$ ) and humidity (np: $21.02 \% \pm 0.17$ and p: $20.12 \% \pm 0.07$ ). Meliponine honey was within the values observed in literature for this type of honey. In most of the physicochemical analysis performed, the types of honey were found to be different from each other, with meliponine honey having higher moisture and water activity, which makes it more susceptible to deterioration. A significant difference was observed between fresh honey from Apis mellifera and meliponine when compared to their respective pasteurized honey for moisture analysis (A. melifera, np: $21.02 \% \pm 0.17$ and p: $20.12 \% \pm 0.07 ; M$. fasciculata, $\mathrm{np}: 24.33 \% \pm 0.03$ and p: $23.68 \% \pm 0.16$; M. flavolineata, np: $28.53 \% \pm 0.02$ and p: 27.40\% \pm 0.04 ), apparent sucrose (A. melifera, np: $5.75 \% \pm 0.18$ and p: $3.19 \% \pm 0.23 ;$ M. fasciculata, np: $3.89 \% \pm 0.04$ and p: $1.59 \% \pm 0.03 ;$ M. flavolineata, np: $2.12 \% \pm 0.22$ and p: $1.62 \% \pm 0.02$ ) and HMF (A. melifera, np: $20.99 \mathrm{mg} / \mathrm{Kg} \pm 0.13$ and p: $64.36 \mathrm{mg} / \mathrm{Kg} \pm 0,07 ;$ M. fasciculata, $\mathrm{np}: 6.54 \mathrm{mg} / \mathrm{Kg} \pm 0.14$ and p: $9.46 \mathrm{mg} / \mathrm{Kg} \pm 0.09$; M. flavolineata, $\mathrm{np}: 3.59 \mathrm{mg} / \mathrm{Kg} \pm 0.14$ and $\mathrm{p}: 43.19 \mathrm{mg} / \mathrm{Kg} \pm 0.14)$. All the samples submitted to the pasteurization process were within the microbiological limits established by current legislation.
\end{abstract}

Keywords: Apis mellifera; characterization; honey; meliponines; pasteurization.

Recebido em: 24 de abril de 2017.

Aceito em: 27 de abril de 2018.

\title{
Introdução
}

A apicultura é uma das atividades mais antigas e importantes do mundo, prestando grande contribuição ao homem através da produção de mel, geleia real, própolis, cera e pólen, bem como na agricultura e nos serviços de polinização ${ }^{(1)}$. Atualmente, a criação de abelhas pode ser dividida em duas práticas: a apicultura e a meliponicultura. A meliponicultura, que é a criação de abelhas sem ferrão, vem ganhando cada vez mais espaço e o mel vem alcançando valores elevados, em virtude de o volume disponível ser menor ${ }^{(2)}$.

A meliponicultura, nome dado à criação de abelhas sem ferrão da subtribo Meliponina, se enquadra perfeitamente dentro dos conceitos de diversificação e uso sustentável da terra, na Amazônia. Os meliponíneos são abelhas dóceis, de fácil manejo e necessitam de pouco investimento para a sua criação, propiciando retorno garantido do investimento com baixo risco $^{(3)}$. É uma atividade que pode ser integrada a plantios florestais, de fruteiras e de culturas de ciclo curto, podendo contribuir, através da polinização, com o aumento da produção agrícola e a regeneração da vegetação natural ${ }^{(4)}$.

O mel de meliponíneos é pouco estudado e a caracterização físico-química desse mel é de suma importância como parte das estratégias de valorização do produto, conferindo identidade e agregação de valor a ele $^{(5)}$. Algumas pesquisas realizadas com esse mel revelaram uma heterogeneidade na sua 
composição de água, açúcares e minerais, que são relacionados à espécie da abelha, origem botânica, região e estado de maturação ${ }^{(6-9)}$.

Dessa forma, devido à crescente expansão do comércio de méis oriundos de abelhas sem ferrão, o estudo das características físico-químicas dos méis mais produzidos no estado do Pará (Melipona flavolineata e Melipona fasciculata) é importante, pois visa conhecer as características de cada um, comparando-os entre si e com o mel tradicional de Apis mellifera L. Os efeitos da pasteurização também devem ser observados nas características físico-químicas desses méis, uma vez que eles são muito perecíveis.

O mel de abelhas do gênero Melipona deve ser submetido a técnicas de conservação, como a pasteurização, visando diminuir a carga microbiana vegetativa e aumentando, assim, sua vida de prateleira, pois o elevado teor de umidade do mel propicia o desenvolvimento microbiológico durante o período de armazenamento ${ }^{(2)}$. Essa prática não é tão comum ao mel de abelhas Apis mellifera L., entretanto, devido à umidade elevada da região amazônica, o mel de Apis mellifera L. pode, em virtude da sua característica higroscópica, apresentar umidade superior ao permitido na legislação específica, proporcionando assim um possível crescimento microbiológico durante o período de estocagem.

Assim, o objetivo do trabalho foi avaliar a qualidade microbiológica e caracterizar físicoquimicamente as amostras de méis in natura e pasteurizadas de duas espécies de abelha sem ferrão (Melipona fasciculata e Melipona flavolineata), assim como o mel de abelhas africanizadas (Apis mellifera) para compará-las entre si.

\section{Material e Métodos}

Foram utilizados méis de abelhas Melipona fasciculata, Melipona flavolineata e Apis mellifera provenientes da Comunidade da Flecheira, Município de Tracuateua, no Nordeste Paraense. As amostras de méis foram pasteurizadas em sistema tubular, montado em escala laboratorial, na qual o binômio aplicado para os méis de Melipona foram $62{ }^{\circ} \mathrm{C} / 60$ s e para o mel de Apis $72{ }^{\circ} \mathrm{C} / 3,5$ min ${ }^{(10)}$.

Umidade: determinada com auxílio de um refratômetro (RTD-45, Instrutherm, São Paulo, Brasil) de acordo com o método $969.38 \mathrm{~B}$ da $\mathrm{AOAC}^{(11)}$.

Atividade de água: utilizando analisador de atividade de água (AQUALAB 4TE, Decagon, Washington, USA) calibrado com soluções saturadas de cloreto de lítio e de magnésio e sulfato de potássio.

Açúcares redutores: determinado por titulometria, seguindo os métodos do CODEX ALIMENTARIUS ${ }^{(12)}$.

Sacarose aparente: determinação dos açúcares redutores, por meio de titulometria, após a inversão por hidrólise ácida, seguindo os métodos do CODEX ALIMENTARIUS ${ }^{(12)}$.

Resíduo mineral fixo: determinado gravimetricamente, por calcinação da amostra a $550{ }^{\circ} \mathrm{C}$, de acordo com o método 900.02 da $\mathrm{AOAC}^{(11)}$.

pH: determinado em potenciômetro (HI9321, Hanna Instruments, Michigan, USA), de acordo com o método do Instituto Adolfo Lutz ${ }^{(13)}$.

Acidez titulável: a acidez total é a soma da acidez livre e lactônica, que são determinadas a partir da titulação utilizando soluções padronizadas de hidróxido de sódio $0,05 \mathrm{~N}$, para a determinação da 
acidez livre; e de ácido clorídrico $0,05 \mathrm{~N}$, para a determinação da acidez lactônica, de acordo com o método 962.19 da $\mathrm{AOAC}^{(11)}$.

Atividade diastásica: pelo método espectrofotométrico, por meio de descoloração de uma solução de amido, iodo e mel em condições controladas, segundo a metodologia do CODEX ALIMENTARIUS ${ }^{(12)}$.

Teor de hidrometilfurfural: pelo método espectrofotométrico conforme o método 980.23 da AOAC $^{(11)}$.

Análise colorimétrica: segundo método de Bianchi ${ }^{(14)}$, no qual a amostra de mel é diluída e é realizada leitura da absorbância em $635 \mathrm{~nm}$. A cor foi expressa em Pfund e calculada através da equação: Cor $=$ $(371,39 \times$ Abs365) - 38,70

Todas as análises foram realizadas em triplicatas e os resultados submetidos à análise estatística, com uma análise de variância (ANOVA) e as médias, quando significativas, foram comparadas pelo teste Tukey, ao nível de 5\% de significância, utilizando o programa XLSTAT (versão 2015.1).

Foram realizadas análises microbiológicas para determinação de Salmonella, coliformes totais e fecais, contagem de bolores e leveduras seguindo os métodos oficiais da APHA - American Public Health Association $^{(15)}$ e os resultados comparados aos padrões estipulados na legislação vigente ${ }^{(16)}$.

\section{Resultados}

Os resultados obtidos nas análises físico-químicas, colorimétricas e microbiológicas são apresentados na Tabela 1, 2 e 3, respectivamente. Os resultados foram analisados segundo a legislação vigente ${ }^{(16,17)}$ e para o mel de meliponíneos foram utilizadas duas literaturas ${ }^{(18,19)}$, as quais propuseram regulamentos para estes méis (Tabela 4).

Tabela 1. Resultados das análises físico-quimicas das amostras de mel analisadas

\begin{tabular}{|c|c|c|c|c|c|c|}
\hline \multirow{2}{*}{ Parâmetros } & \multicolumn{2}{|c|}{ M. fasciculata } & \multicolumn{2}{|c|}{ M. flavolineata } & \multicolumn{2}{|c|}{ A. mellifera } \\
\hline & np & $\mathbf{P}$ & np & $\mathbf{P}$ & np & $\mathbf{p}$ \\
\hline $\mathrm{U}$ & $24,33^{\mathrm{c}} \pm 0,03$ & $23,68^{d} \pm 0,16$ & $28,53^{a} \pm 0,02$ & $27,40^{b} \pm 0,04$ & $21,02^{f} \pm 0,17$ & $20,12^{\mathrm{e}} \pm 0,07$ \\
\hline & $84^{\circ} \pm$ & $0,6813^{d} \pm$ & $459^{\mathrm{a}} \pm$ & $0,7313^{b} \pm$ & $0,6406^{e} \pm$ & $0^{f} \pm$ \\
\hline & 0,0007 & 0,0003 & 0,0012 & 0,0006 & 0,0004 & 0,0 \\
\hline A & $63,47^{a} \pm 0,24$ & $63,83^{\mathrm{a}} \pm 2,37$ & $63,09^{\mathrm{a}} \pm 1,55$ & $62,70^{\mathrm{a}} \pm 0,19$ & $57,43^{b} \pm 1,28$ & $58,53^{b} \pm 1,91$ \\
\hline SA ( & $3,89^{b} \pm 0,04$ & $1,59^{\mathrm{e}} \pm 0,03$ & $2,12^{d} \pm 0,22$ & $1,62^{e} \pm 0,02$ & $5,75^{a} \pm 0,18$ &, 23 \\
\hline $\mathrm{RM}(\%)$ & $0,21^{\mathrm{a}, \mathrm{b}} \pm 0,02$ & $0,19^{b} \pm 0,02$ & $0,25^{a, b} \pm 0,00$ & $0,25^{\mathrm{a}} \pm 0,03$ & $0,11^{\mathrm{c}} \pm 0,01$ & $0,11^{\mathrm{c}} \pm 0,01$ \\
\hline $\mathrm{pH}$ & $4,59^{a} \pm 0,02$ & $4,87^{a} \pm 0,06$ & $4,28^{a, b} \pm 0,03$ & $4,46^{a} \pm 0,09$ & $3,46^{b, c} \pm 0,03$ & $3,42^{c} \pm 0,03$ \\
\hline $\begin{array}{c}\mathrm{AT} \\
(\mathrm{cmol} / \mathrm{kg})\end{array}$ & $17,64^{c} \pm 0,75$ & $16,08^{\mathrm{c}} \pm 1,39$ & $38,85^{b} \pm$ & $32,40^{\circ} \pm 1,19$ & $62,25^{2} \pm 4,15$ & $62,67^{a} \pm 5,75$ \\
\hline $\mathrm{AD}$ (Gothe) & Não & do & $6,31^{\mathrm{c}} \pm 0,09$ & $5,75^{c} \pm 0,13$ & $24,83^{a} \pm 0,17$ & $20,56^{\mathrm{b}} \pm 1,45$ \\
\hline $\mathrm{HMF}(\mathrm{mg} / \mathrm{kg})$ & $6,54^{\mathrm{e}} \pm 0,14$ & $9,46^{d} \pm 0,09$ & $3,59^{f} \pm 0,14$ & $43,10^{b} \pm 0,85$ & $20,99^{c} \pm 0,13$ & $64,36^{a} \pm 0,07$ \\
\hline
\end{tabular}

Resultados expressos como média \pm desvio padrão. Médias com letras iguais na mesma linha não diferem significativamente de acordo com o teste de Tukey a $\mathrm{p} \leq 0,05$.

Onde, np: não pasteurizado; p: pasteurizado; U: umidade; Aa: atividade de água; AR: açúcares redutores; AS: sacarose aparente; $\mathrm{RM}$ : resíduo mineral; $\mathrm{AT}$ : acidez titulável; $\mathrm{AD}$ : atividade diastásica; $\mathrm{HMF}$ : hidroximetilfurfural. 
Tabela 2. Resultados da análise de cor das amostras de mel

\begin{tabular}{ccc}
\hline \multicolumn{2}{c}{ Amostra } & Cor \\
\hline \multirow{2}{*}{ M. fasciculata } & Não pasteurizada & Âmbar claro \\
& Pasteurizada & Âmbar claro \\
M. flavolineata & Não pasteurizada & Âmbar \\
& Pasteurizada & Âmbar \\
A. mellifera & Não pasteurizada & Âmbar escuro \\
& Pasteurizada & Âmbar escuro \\
\hline
\end{tabular}

Tabela 3. Análises microbiológicas nas amostras de mel e padrões estabelecidos pela legislação

\begin{tabular}{|c|c|c|c|}
\hline Amostra & $\begin{array}{c}\text { Salmonella } \\
\text { spp }\end{array}$ & $\begin{array}{c}\text { Coliformes } \\
\text { totais }\end{array}$ & Fungos e leveduras \\
\hline M. fasciculata sem pasteurização & Ausente & $<3 \mathrm{NMP} / \mathrm{g}$ & $<1 \times 10^{:} \mathrm{UFC} / \mathrm{g}$ \\
\hline M. fasciculata pasteurizada & Ausente & $<3 \mathrm{NMP} / \mathrm{g}$ & $<1 \times 10^{:} \mathrm{UFC} / \mathrm{g}$ \\
\hline M. flavolineata sem pasteurização & Ausente & $>1100 \mathrm{NMP} / \mathrm{g}$ & $1,19 \times 10^{6} \mathrm{UFC} / \mathrm{g}$ \\
\hline M. flavolineata pasteurizada & Ausente & $<3 \mathrm{NMP} / \mathrm{g}$ & $<1 \times 10^{:} \mathrm{UFC} / \mathrm{g}$ \\
\hline A. mellifera sem pasteurização & Ausente & $<3 \mathrm{NMP} / \mathrm{g}$ & $<1 \times 10^{:} \mathrm{UFC} / \mathrm{g}$ \\
\hline A. mellifera pasteurizada & Ausente & $<3 \mathrm{NMP} / \mathrm{g}$ & $<1 \times 10^{:} \mathrm{UFC} / \mathrm{g}$ \\
\hline MERCOSUL ${ }^{(16)}$ & Ausente & $<3 \mathrm{NMP} / \mathrm{g}$ & $10^{2} \mathrm{UFC} / \mathrm{g}$ \\
\hline
\end{tabular}

Tabela 4. Parâmetros físico-químicos estabelecidos pela legislação brasileira para análise de mel de Apis mellifera e parâmetros físico-químicos propostos para mel de abelhas do gênero Melipona.

\begin{tabular}{cccc}
\hline & $\begin{array}{c}\text { Mel de } \text { A. } \\
\text { mellifera }\end{array}$ & \multicolumn{2}{c}{ Mel de melípona } \\
\hline Parâmetro & BRASIL ${ }^{(17)}$ & $\begin{array}{c}\text { Villas-Bôas e } \\
\text { Malaspina }\end{array}$ & Carvalho et al. (19) \\
& & Máx. 35 & Máx. 30 \\
\hline Umidade (\%) & Máx. 20 & Mín. 50 & Mín. 60 \\
Açúcares redutores (\%) & Mín. 65 & Máx. 6 & Máx. 6 \\
Sacarose aparente (\%) & Máx. 6 & Máx. 0,4 & Máx. 0,1 \\
Sólidos insolúveis (\%) & Máx. 0,1 & Máx. 0,6 & Máx. 0,6 \\
Minerais (\%) & Máx. 0,6 & Máx. 85 & Máx. 50 \\
Acidez (mEq/Kg) & Máx. 50 & Mín. 0,3 & Máx. 3 \\
İndice de diástase (Gothe) & Mín. 8 & Máx. 40 & Máx. 10 \\
HMF (mg/Kg) & Máx. 60 & &
\end{tabular}

\section{Discussão}

Na Tabela 1, observou-se que todas as amostras diferiram entre si e que o processo de pasteurização provocou uma redução da umidade do mel. Entre as amostras estudadas, o mel de A. mellifera foi o 
que apresentou valores de umidade menores.

O mel de meliponídeos apresenta teores de umidade superiores aos encontrados para mel de Apis melifera, isso ocorre pelo modo como as abelhas operculam o mel, que é diferente entre os gêneros; de modo geral, abelhas do gênero Apis operculam quando o mel apresenta umidade entre 17-18\%, embora essa umidade possa ser alterada de acordo com a umidade do local onde o mel é produzido; enquanto que abelhas do gênero Melipona operculam o mel quando a umidade é de cerca de $24 \%{ }^{(20)}$.

Segundo a legislação brasileira para mel de A $_{\text {mellifera }}{ }^{(1)}$, a umidade máxima permitida é $20 \%$. O mel de Apis apresentou valores de $20,12 \%$ e $21,02 \%$, superior ao permitido pela legislação, no entanto, outros autores também observaram valores superiores ao permitido pela legislação. Esses valores de umidade superiores podem ser explicados pela característica higroscópica dos méis ${ }^{(21)}$, que deve ser considerada quando o mel é produzido em um ambiente com umidade relativa superior a do mel, variando de 75 a $90 \%$ ao longo do ano, como a região amazônica ${ }^{(22)}$.

Apesar de não existir legislação para o mel de meliponíneos, as amostras encontram-se dentro dos valores propostos por Carvalho ${ }^{(19)}$ e Villas-Bôas e Malaspina ${ }^{(18)}$, máximo de $30 \%$ e $35 \%$ de umidade, respectivamente.

Em estudo sobre amostras de mel de duas espécies de meliponíneos (Scaptotrigona sp. e Tetragonisca angustula, oriundas de Belterra no oeste do Pará e Angra dos Reis no Rio de Janeiro, respectivamente) e de mel de laranja de Apis mellifera (oriundas do Rio de Janeiro, Minas Gerais e São Paulo), os valores médios encontrados foram de 17,14\% para mel de Apis mellifera, 27,15\% para mel de Scaptotrigona sp. e 29,00\% para mel de $T$. angustula ${ }^{(23)}$. Os valores deste estudo foram inferiores para mel de $A$. mellifera e semelhantes para mel meliponíneo.

O mel de M. fasciculata oriundo do estado do Maranhão apresentou umidade entre 21,44 a 27,51\%, semelhantes às encontradas neste trabalho para mel de abelhas da mesma espécie ${ }^{(24)}$.

Comparando o mel de M. fasciculata e M. flavolineata estudados neste trabalho, com mel de outras espécies de meliponíneos, observa-se valores semelhantes. Foram analisadas 152 amostras de mel de diferentes espécies de meliponíneos e encontraram umidade entre 19,9 e 41,9\%\%

Foram avaliadas três amostras de mel de abelhas do gênero Melipona da Amazônia equatoriana com umidade de $34,1 \% \pm 4,34^{(26)}$, superior ao encontrado neste trabalho. Em outro estudo analisando mel de Apis mellifera, M. beecheii e Trigona spp, encontraram umidade de 19,0\%, 28,2\% e 25,0\%, respectivamente ${ }^{(27)}$.

Acompanhando o observado aos teores de umidade, todas as amostras de mel igualmente diferiram significativamente entre si, indicando que a água livre nas amostras de mel está relacionada com a espécie de abelha. $\mathrm{O}$ mel de abelhas do gênero Melipona apresentou valores de atividade de água superiores aos encontrados em mel de $A$. mellifera.

$\mathrm{Na}$ literatura também se observa isso. Foram encontrados valores de 0,540 a 0,60 em mel de Apis mellifera L. ${ }^{(28)}$, inferiores ao encontrado neste trabalho para mel de abelhas da mesma espécie.

No estudo de mel de abelha do gênero Melipona, encontraram valores de atividade de água de 0,740 a $0,760^{(6)}$; outros autores encontraram uma maior variação, entre 0,662 e $0,851^{(29)}$ e também entre 0,650 e 0,720 para mel de M. subnitida coletadas no estado da Paraíba ${ }^{(9)}$. 
Neste trabalho, embora a aplicação do tratamento térmico (pasteurização) tenha gerado valores inferiores de atividade de água, indicando perdas significativas pelo teste estatístico aplicado (em função dos desvios serem muito baixos uma vez que a medição é feita em equipamento digital), essa redução foi discreta para o mel de $M$. flavolineata e $A$. mellifera e praticamente inexistente para $M$. fasciculata, não tendo essa diminuição importância em termos tecnológicos.

A água presente no mel apresenta forte interação com as moléculas dos açúcares, diminuindo a disponibilidade das moléculas de água para o desenvolvimento microbiológico ${ }^{(30)}$, permitindo avaliar a suscetibilidade de deterioração dos alimentos e, consequentemente, a vida de prateleira do produto.

Por apresentarem valores de atividade de água elevados, os méis de meliponíneos são mais suscetíveis a contaminação, necessitando de maior cuidado no manuseio e armazenamento, sendo indispensável a utilização de técnicas como a refrigeração, desumidificação ou pasteurização ${ }^{(31)}$.

Em relação aos açúcares redutores, observou-se que o processo de pasteurização não influenciou no teor das amostras estudadas, no entanto, o mel de $A$. mellifera apresentou valores significantemente inferiores ao mel de melipona.

O mel de $A$. mellifera apresentou valores inferiores ao estipulado pela legislação brasileira, na qual os açúcares redutores devem ser no mínimo $65 \%{ }^{(17)}$. Outros autores afirmam que valores abaixo de $65 \%$ podem indicar um mel não amadurecido para colheita e consequentemente com umidade elevada ${ }^{(32)}$.

Diferentemente do mel de $A$. mellifera, o mel de abelhas do gênero Melipona apresenta açúcares redutores em concentrações menores, por isso os valores mínimos propostos por Carvalho ${ }^{(19)}$ e VillasBôas e Malaspina ${ }^{(18)}$ para açúcares redutores presentes em mel de meliponíneos são respectivamente $60 \%$ e $50 \%$. Assim, as amostras estudadas enquadram-se dentro do proposto pelos autores.

Outros autores determinaram teores de açúcares redutores variando de 58,14\% até 61,35\% para mel de S. bipunctata $^{(33)}$. Em outro estudo, avaliaram três amostras de mel de abelhas do gênero Melipona da Amazônia equatoriana e encontraram valores médios de açúcares redutores de 44,9\% ${ }^{(26)}$. Em outro estudo, os autores encontraram valores médios de açúcares redutores de 60,68\% para mel de $M$. fasciculata da região do cerrado maranhense, valores estes inferiores aos observados no mel

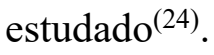

Foram analisadas 152 amostras de mel de diferentes espécies de meliponíneos e obteve-se valores entre 58,00\% e 75,7\% para açúcares redutores ${ }^{(25)}$. Analisando nove amostras de mel de $M$. subnitida, coletadas no estado da Paraíba, os autores encontraram valores entre 50,50\% e 72,77\% ${ }^{(9)}$.

Em relação à sacarose aparente, todo o mel analisado está dentro dos padrões estabelecidos, cujo máximo permitido é de $6 \%$. Apesar do mel de $A$. mellifera não estar dentro do proposto pela legislação para o teor de açúcares redutores, ele se apresentou dentro do limite para sacarose aparente, excluindo a possibilidade de adulteração do mel. Observa-se ainda que os teores de sacarose aparente foram influenciados pelo processo de pasteurização, ocorrendo diminuição significativa do teor de sacarose do mel in natura quando comparado ao mel pasteurizado.

Os valores observados neste trabalho são semelhantes ao encontrados por outros autores, os quais, ao avaliar mel de Melipona fasciculata, encontraram valores de sacarose aparente variando de 0,89 a $6,08 \%{ }^{(24)}$. Em outro trabalho, analisaram 152 amostras de mel de diferentes espécies de meliponíneos 
e obtiveram valores entre $1,10 \%$ e $4,80 \%$ para sacarose aparente ${ }^{(25)}$. Enquanto que foi determinado os teores de sacarose aparente em 75 amostras de mel de Apis mellifera de algumas regiões de Portugal e encontraram valores entre 2,6 e $2,9 \%{ }^{(34)}$.

Quanto ao resíduo mineral fixo avaliado nas amostras de mel, igualmente observou-se que todos se encontraram dentro do estabelecido pela legislação.

A pasteurização não influenciou no teor de resíduo mineral das amostras, porém houve diferença $(\mathrm{p} \leq 0,05)$ entre mel de gêneros diferentes, sendo que os teores de resíduo mineral nos méis de $A$. mellifera foram significativamente inferiores aos demais.

Outros autores determinaram teores de resíduo mineral para mel de A. mellifera e S. bipunctata, de $0,25 \%$ até $0,56 \%$ e $0,79 \%$ até $0,88 \%{ }^{(33)}$, respectivamente. Comparando com o trabalho, os valores mostraram-se semelhantes para o mel de A. mellifera, entretanto, os teores de resíduo mineral presentes nas amostras de mel do referido autor foram superiores. Em mel de A. mellifera, outros autores encontraram teor médio de cinzas de $0,14 \%{ }^{(35)}$.

O teor de resíduo mineral, é um indicativo de possíveis irregularidades no mel, como, por exemplo, a falta de higiene e a não decantação e/ ou filtração no final do processo de retirada do mel pelo apicultor $^{(36)}$.

Em relação ao $\mathrm{pH}$, o mel é um produto normalmente ácido, com $\mathrm{pH}$ variando entre $3,2-4,5^{(1)}$, no entanto a legislação brasileira não estabelece limites para valores de $\mathrm{pH}$ em mel, mas a determinação desse parâmetro é útil para a determinação da qualidade.

Pode-se observar que a pasteurização não influenciou significativamente no $\mathrm{pH}$ dos méis. Entretanto, o mel de A. mellifera apresentou valor de $\mathrm{pH}$ significantemente inferior aos méis de meliponíneos.

Outros autores, ao analisarem os valores de $\mathrm{pH}$ em amostras de mel, também encontraram valores semelhantes aos observados neste trabalho. Em estudo sobre 39 amostras de mel de Portugal, com diferentes origens florais, autores relataram valores entre 3,3 e $4,4^{(37)}$. Em outro estudo analisando 152 amostras de mel de diferentes espécies de meliponíneos obtiveram valores de pH entre 3,15 e $4,66^{(25)}$.

Para a acidez total, a legislação brasileira estabelece limites para mel de abelhas do gênero Apis mellifera de no máximo $50 \mathrm{cmol} \mathrm{kg}^{-1(17)}$. Observa-se que o mel de Apis mellifera apresentou valores superiores ao estabelecido pela legislação vigente.

O mel de meliponíneos apresentou acidez titulável dentro do proposto por Carvalho ${ }^{(19)} \mathrm{e}$ Villas-Bôas e Malaspina ${ }^{(18)}$ para mel de meliponíneos, que são no máximo $50 \mathrm{cmol} \mathrm{kg}{ }^{-1}$ e $85 \mathrm{cmol} \mathrm{kg}^{-1}$, respectivamente.

Os valores de acidez total nas amostras de mel não foram influenciados pelo processo de pasteurização. Observa-se também que existe diferença significativa $(p \leq 0,05)$ nos valores de acidez entre as amostras de mel de diferentes espécies de abelha. Valores de acidez elevados podem estar relacionados à características próprias de mel da região e à composição do néctar das flores; no entanto valores excessivamente elevados de acidez podem indicar proliferação microbiana ${ }^{(38)}$.

Os valores encontrados neste trabalho estão condizentes com valores encontrados por outros autores. Ao analisar os valores de acidez total de 39 amostras de mel originárias de Portugal, com diferentes 
origens florais, foram relatados valores de 17,7 até $70,5 \mathrm{cmol} \mathrm{kg}^{-1(37)}$. Já outros autores analisaram 152 amostras de mel de diferentes espécies de meliponíneos e obtiveram valores de acidez entre 5,9 e $109,0 \mathrm{cmol} \mathrm{kg}^{-1(25)}$.

Em outro estudo, analisando amostras de mel de Apis mellifera, M. beecheii e Trigona spp outros autores encontraram valores de acidez de $37,3 \mathrm{cmol} \mathrm{kg}{ }^{-1}$, 38,6 cmol kg-1 e 36,2 $\mathrm{cmol} \mathrm{kg}^{-1}$, respectivamente ${ }^{(27)}$. Analisando a acidez de 9 amostras de mel de M. subnitida, coletadas no Estado da Paraíba, encontraram valores entre 24,66 e 59,66 $\mathrm{cmol} \mathrm{kg}^{-1}(9)$.

Quanto à atividade diastásica, por ser um indicativo da qualidade do mel, a legislação brasileira para mel de Apis mellifera estabelece valor mínimo de 8 na escala Gothe, se o teor de HMF for inferior a $15 \mathrm{mg} \mathrm{kg}^{-1}$, a legislação admite valor de atividade diastásica mínima de $3^{(17)}$, enquanto que para o mel de meliponíneos foram propostos valores de no máximo 3 e no mínimo 0,3 na escala Gothe, por Carvalho $^{(19)}$ e Villas-Bôas e Malaspina ${ }^{(18)}$, respectivamente.

A metodologia utilizada não foi eficiente para a determinação da atividade diastásica nas amostras de mel de $M$. fasciculata, possivelmente por conter quantidade muito baixa da enzima. Entretanto, para as amostras de mel de $M$. flavolineata a metodologia foi adequada, sendo possível observar que a pasteurização provocou uma redução da atividade diastásica, porém ela não foi significativa $(\mathrm{p} \leq 0,05)$.

Para o mel de $A$. mellifera, os valores da atividade foram bem superiores aos observados para $M$. flavolineata e a pasteurização apresentou influência significativa, com redução do valor da atividade em 17,19\% após o processo térmico.

Esse comportamento pode ser explicado pela diferença na temperatura da pasteurização do mel, que foram $62{ }^{\circ} \mathrm{C}$ para os méis de M. flavolineata e $72{ }^{\circ} \mathrm{C}$ para os méis de A. mellifera, uma vez que a atividade da enzima é influenciada com a temperatura, diminuindo com aquecimentos moderados ou elevados.

Quanto à legislação, o mel de Apis mellifera estudado está de acordo com o estabelecido, assim como as amostras de mel de Melipona flavolineata estão condizentes com o que é proposto por Villas-Bôas e Malaspina ${ }^{(18)}$, entretanto apresentou-se superior ao proposto por Carvalho ${ }^{(19)}$, que é no máximo 3. Segundo Carvalho ${ }^{(19)}$, pesquisas demonstram que mel de meliponíneos apresenta atividade diastásica inferior ao estabelecido pela legislação para Apis, mesmo em amostras de mel recém-coletadas e sem sofrerem aquecimento.

Entretanto, a literatura indica uma grande variação nos valores de atividade diastásica em mel de melíponas, por exemplo, em mel de Melipona fasciculata Smith da região do cerrado maranhense encontraram índices de diástase variando de 0,60 a 2,93 Gothe ${ }^{(24)}$, outros autores analisando 152 amostras de mel de diferentes espécies de meliponíneos obtiveram valores entre 0,9 e 23,0 na escala Gothe $^{(25)}$.

Em relação ao teor de hidroximetilfurfural, composto resultante da transformação dos açúcares frutose e glicose, que estão presentes naturalmente no mel, observou-se que o processo de pasteurização das amostras influenciou significativamente na formação do composto e que o teor inicial do mesmo, entre as diferentes amostras de mel, também apresentou diferença significativa.

A legislação brasileira estabelece $60 \mathrm{mg} \mathrm{kg}^{-1}$ como limite máximo para HMF em mel ${ }^{(17)}$. A amostra de $A$. mellifera pasteurizada encontra-se com teor superior ao permitido pela legislação brasileira. 
Entretanto, convém ressaltar que a legislação brasileira não estipula parâmetros para mel pasteurizado. Dentre os méis de meliponíneos, apenas a amostra de mel de M. flavolineata pasteurizada apresentou valor superior ao proposto por Carvalho ${ }^{(19)}$ e Villas-Bôas e Malaspina ${ }^{(18)}$, os quais propuseram limites máximos de $10 \mathrm{mg} \mathrm{kg}^{-1} \mathrm{e} 40 \mathrm{mg} \mathrm{kg}^{-1}$, respectivamente.

O Codex Alimentarius ${ }^{(12)}$ estabelece um limite de $80 \mathrm{mg} \mathrm{kg}^{-1}$ para mel proveniente de países tropicais, em virtude de que, em países quentes, o teor de HMF tende a ser superior e aumentar mais rápido no período de armazenamento, já que o HMF é um composto formado quando o mel é submetido a temperaturas elevadas. Em outro estudo, no qual avaliaram o teor de HMF em 12 amostras de mel de A. mellifera no município de Apodi no estado do Rio Grande do Norte e obtiveram valores elevados quando comparados com o mel estudado neste trabalho, entre 70,65 e 150,27 mg kg-1(39).

Outros autores, ao estudarem mel de Melipona Illiger da região nordeste do Brasil, encontraram valores de 0 a 60,2 $\mathrm{mg} \mathrm{kg}^{-1(29)}$. Em outro estudo, foram relatados valores entre 6,08 a 194,74 $\mathrm{mg} \mathrm{kg}^{-1}$ para mel de abelhas $A$. mellifera e valores entre 4,20 a 5,80 $\mathrm{mg} \mathrm{kg}^{-1}$ para mel de abelhas sem ferrão $S$. bipunctata $^{(33)}$. Semelhante ao encontrado neste trabalho, o teor de HMF é superior em mel de $A$. mellifera quando comparado com o teor presente em mel de meliponíneos. Assim como Lira ${ }^{(23)}$, ao compararem mel de $A$. mellifera e meliponíneos, encontraram valores entre 7,49 a 56,80 $\mathrm{mg} \mathrm{kg}^{-1}$ para mel de $A$. mellifera e 0,46 a 7,49 $\mathrm{mg} \mathrm{kg}^{-1}$ para as de abelhas nativas.

Quanto à cor dos méis estudados, foram observadas diferentes colorações (Tabela 2). De acordo com a legislação vigente, a cor do mel pode variar desde o branco-água até o âmbar-escuro ${ }^{(17)}$, característica que varia de acordo com a espécie das abelhas, condições climáticas e origem florística. Nos méis estudados, a coloração variou do âmbar claro para o mel de M. fasciculata; âmbar, para o mel de $M$. flavolineata e âmbar escuro para o mel de A. mellifera. Ressaltamos que não houve alteração da cor do mel após o processo de pasteurização.

Os resultados encontrados por outros autores mostram coloração semelhante aos deste estudo, ao avaliarem a cor de mel de Melipona fasciculata da região do cerrado maranhense, em outro estudo obteve-se grande variedade de cores, predominando o extra âmbar claro e âmbar claro ${ }^{(24)}$. Foi encontrado também, ao avaliarem mel de Apis mellifera oriundo da Malásia, as cores variaram do âmbar claro ao âmbar escuro ${ }^{(40)}$.

Por fim, a Tabela 3 permitiu avaliar a qualidade higiênico-sanitária do mel avaliado. Para a comparação dos resultados microbiológicos, utilizou-se o regulamento técnico do MERCOSUL ${ }^{(16)}$ para identidade de qualidade do mel, o qual estabelece limites para Salmonella spp, coliformes totais e fungos e leveduras. Exceto a amostra de mel de $M$. flavolineata sem pasteurização que apresentou contaminação para coliformes e fungos e leveduras, as demais se apresentaram dentro dos limites estabelecidos pela legislação, para todas as análises.

A ausência de micro-organismos em quase todas as amostras pode ser explicada pela acidez do mel, que inibe o crescimento dos micro-organismos, apesar da elevada taxa de umidade ${ }^{(24)}$. O mel de $M$. flavolineata sem pasteurização mostrou-se fora do estipulado pela legislação em função do elevado valor de atividade de água e, provavelmente, por um manuseio inadequado no momento da colheita ou envase e armazenamento do mel durante o transporte. Entretanto, a pasteurização realizada foi eficiente para redução da carga microbiana, tornando o mel apto ao consumo. 


\section{Conclusão}

Os méis de A. mellifera, M. fasciculata e M. flavolineata mostraram-se diferentes entre si em quase todas as análises físico-químicas, sendo que os méis de meliponíneos apresentam umidade e atividade de água mais elevada. Observou-se que o processo de pasteurização influenciou de forma significativa a umidade, $\mathrm{pH}$, sacarose aparente e HMF dos méis, porém não influenciou nas características físico-químicas de acidez, cinzas e açúcares redutores. A exceção das amostras de mel de M. flavolineata sem pasteurização, as demais se apresentaram dentro dos limites microbiológicos estabelecidos pela legislação. O processo de pasteurização se mostrou eficaz na redução dos microorganismos presentes nas amostras de M. flavolineata.

\section{Agradecimentos}

Conselho Nacional de Desenvolvimento Científico e Tecnológico (CNPq) pela concessão da bolsa de mestrado do primeiro autor.

\section{Referências}

1. Crane E. Honey, A comprehensive survey. 3rd ed. Londres: Willian Heinemann. 1979. 608p. Inglês.

2. Camargo, RCR. de; Oliveira, KL. de; Berto, MI. Mel de abelhas sem ferrão: proposta de regulamentação. Brazilian Journal of Food Technology. 2017. Versão online. Campinas. v. 20, e2016157. Doi:10.1590/19816723.15716.

3. Slaa EJ, Sánchez-Chaves LA, Malagodi-Braga KS, Hofstede FE. Stingless bees in applied pollination: practice and perspectives. Apidologie. 2006 Mar./Abr. 37(2):293-315.

4. Giannini T, Boff S, Cordeiro G, Cartolano JE, Veiga A, Imperatriz-Fonseca V, Saraiva AM. Crop pollinators in Brazil: a review of reported interactions. Apidologie, 2015 Mar. 46(2):209-223.

5. Bendini JN, Souza DC. Caracterização físico-química do mel de abelhas proveniente da florada do cajueiro. Ciência Rural, 2008 Mar./Abr. 38(2):565-567.

6. Almeida-Muradian LB, Matsuda AH, Bastos DHM. Physicochemical parameters of Amazon Melipona honey. Química Nova, 2007 Mai./Jun. 30(3):707-708.

7. Almeida-Anacleto D, Souza BA, Marchini LC, Moreti ACCC. Composição de amostras de mel de abelha Jataí (Tetragonisca angustula latreille, 1811). Ciência e Tecnologia de Alimentos, 2009 Jul./Set. 29(3):535541.

8. Vit P, Deliza R, Pérez A. How a huottuja (Piaroa) community perceives genuine and false honey from the Venezuelan Amazon, by free-choice profile sensory method. Brazilian Journal of Pharmacognosy, 2011 Jun. 21(5):786-792.

9. Silva TMS, Santos FP Dos, Evangelista-Rodrigues A, Silva EMS Da, Silva GS Da, Novais JS De, Santos F De AR Dos, Camara CA. Phenolic compounds, melissopalynological, physicochemical analysis and antioxidante activity of jandaíra (Melipona subnitida) honey. Journal of Food Composition and Analysis. 2013 
Fev. 29(1):10-18.

10. Nogueira-Neto P. Vida e criação de abelhas indígenas sem ferrão. São Paulo: ed. Nogueirapis, 1997. 446p.

11. AOAC. Official Methods of Anaysis of the Association of Official Analytical Chemists, $16^{\text {th }}$. Ed. Arlington: AOAC. 1995.

12. Codex Alimentarius Commission. CAC/VOL III, Suppl. 2. Ed. 1. Rome: FAO/WHO, 1989.

13. Instituto Adolfo Lutz. Métodos Físico-Químicos para Análise de Alimentos. 4 ed. São Paulo. 2004. 1032p. Português.

14. Bianchi EM. La miel, características y composición - Análisis y Adulteraciones. Santiago del Estero: UNSE - CEDIA, 1981.

15. Vanderzant C, Splittstoesser DF. Compendium of methods for microbiological examination of foods. 3.ed. American Public Health Association. Washington, DC. 1992. 914p.

16. Mercosul. Regulamento técnico MERCOSUL de identidade e qualidade do mel. Resolução ${ }^{\circ}{ }^{\circ} 15.1994$. Português.

17. Brasil - Ministério da Agricultura e do Abastecimento. Instrução Normativa $n^{0} 11$, de 20 de outubro de 2000. Regulamento Técnico de Identidade e Qualidade do Mel. Diário Oficia da União. 2000 Out. 23. Português.

18. Villas-Bôas JK, Malaspina O. Parâmetros físico-químicos propostos para o controle de qualidade do mel de abelhas sem ferrão no Brasil. Revista Mensagem Doce, 2005. 82 (2): 6-16.

19. Carvalho CAL, Alves RMO, Souza BA, Véras SO, Alves EM, Sodré GSM. Proposta de regulamento técnico de qualidade fisico-química do mel floral processado produzido por abelhas do gênero Melipona. pp. 1-9. En Vit P \& Roubik DW, eds. Stingless bees process honey and pollen in cerumen pots. 2013. Facultad de Farmacia y Bioanálisis, Universidad de Los Andes; Mérida, Venezuela. $<$ http://www.saber.ula.ve/handle/123456789/35292>. Acesso em 14 de março de 2016.

20. Evangelista-Rodrigues A, Silva EMS Da, Beserra EMF, Rodrigues ML. Análise físico-química dos méis das abelhas Apis mellifera e Melipona scutellaris produzidos em regiões distintas no Estado da Paraíba. Ciência Rural, 2005 Set./Out. 35(5):1166-1171.

21. Mendes, C. De G.; Da Silva, JBA.; De Mesquita, LX.; Maracajá, PB. As Análises de Mel: Revisão. Caatinga (Mossoró,Brasil), 2009. 22(2):07-14.

22. Almeida, R.; Zanlorenssi, G. Como é a umidade do ar nas capitais ao longo do ano. Jornal Nexo. 2017. Disponível em: https://www.nexojornal.com.br/grafico/2017/09/25/Como-\%C3\%A9-a-umidade-do-ar-nascapitais-ao-longo-do-ano. Acesso em: 25 de abril de 2018.

23. Lira AF, Sousa JPL De, Lorenzon MCA, Vianna CAFJ, Castro RN. Estudo comparativo do mel de Apis mellifera com méis de meliponíneos de diferentes regiões. Acta Veterinária Brasilica, 2014. 8(3):169-178.

24. Holanda CA, Oliveira AR, Costa CP, Ribeiro MNS, Souza JL, Araújo MJAM. Qualidade dos méis produzidos por Melipona fasciculata Smith da região do cerrado maranhense. Química Nova, 2012 Jul. 35(1):55-58.

25. Souza B, Roubik D, Barth O, Heard T, Enríquez E, Carvalho C, Villas-Bôas J. Marchini L, Locatelli J, Persano-Oddo L, Almeida-Muradian L, Bogdanov S, Vit P. Composition of stingless bee honey: setting quality standards. Interciencia, 2006 Dez. 31(12):867 - 882.

26. Guerrini A, Bruni R, Maietti S, Poli F, Rossi D, Paganetto G, Muzzoli M, Scalvenzi L, Sacchetti G. Ecuadorian stingless bee (Meliponinae) honey: A chemical and functional profile of an ancient health product. 
Food Chemistry, 2009 Jun. 114(4):1413-1420.

27. Ramón-Sierra JM, Ruiz-Ruiz JC, Ortiz-Vázquez E de la L. Electrophoresis characterization of protein as a method to establish the entomological origin of stingless bee honeys. Food Chemistry, 2015 Set. 183(15):4348.

28. Schlabitz C, Silva SAF, Souza CFV. Avaliação de parâmetros físico-químicos e microbiológicos em mel. Revista Brasileira de Tecnologia Agroindustrial, 2010. 4(1):80-90.

29. Souza BA, Marchini LC, Odar-Souza M, Carvalho CAL, Alves RMO. Caracterização do mel produzido por espécies de Melipona Illiger, 1806 (Apidae: Meliponini) da região nordeste do Brasil. Química Nova, 2009. 32(2):303-308.

30. Abramovic H, Jamnik M, Burkan L, Kac M. Water activity and water content in Slovenian honeys. Food Control, 2008 Nov.; 19(11):1086-1090.

31. Venturieri GC. Criação de abelhas indígena sem ferrão. 2 ed. Belém-PA. Embrapa Amazônia Oriental, 2008. 60p.

32. Mendonça K, Marchini LC, Souza BA, Almeida-Anacleto D, Moreti ACCC. Caracterização físico-química de amostras de méis produzidas por Apis mellifera L. em fragmento de cerrado no município de Itirapina, São Paulo. Ciência Rural, 2008 Set. 38(6):1748-1753.

33. Oliveira ENA, Santos DC. Análise físico-química de méis de abelhas africanizada e nativa. Revista Instituto Adolfo Lutz, 2011 Abr./Jun. 70(2):132-138.

34. Estevinho ML, Feás X, Seijas JA, Vázquez-Tato MP. Organic honey from Trás-Os-Montes region (Portugal): Chemical, palynological, microbiological and bioactive compounds characterization. Food and Chemical Toxicology, 2012 Fev. 50(2):258-264.

35. Abadio-Finco FDB, Moura LL, Silva IG. Propriedades físicas e químicas do mel de Apis mellifera L. Ciência Tecnologia de Alimentos. 2010 Jul/Set; 30(3):706-12.

36. Vilhena F, Almeida-Muradian LB. Análises físico-químicas de méis de São Paulo. Revista Mensagem Doce. 1999. 53:17-9.

37. Alves A, Ramos A, Gonçalves MM, Bernardo M, Mendes B. Antioxidante activity, quality parameters and mineral contente of Portuguese monofloral honeys. Journal of Food Composition and Analysis. 2013 Jun. 30(2):130-138.

38. Bertoldi FC, Reis VDA, Gonzaga LV, Congro CR. Caracterização físico-química e sensorial de amostras de mel de abelhas africanizadas (Apis mellifera L.) produzidas no pantanal. Evidência: Biotecnologia e Alimentos, 2007 Jan./Jun. 7(1):63-74.

39. Soares KM De P, Aroucha EMM, Góis VA De. Qualidade físico-química de méis silvestres comercializados no município de Apodi, RN. Acta Veterinaria Basílica, 2010. 4(1):55-58.

40. Chua LS, Abdul-Rahaman N, Sarmidi MR, Aziz R. Multi-elemental composition and physical properties of honey samples from Malaysia. Food Chemistry, 2010 Dez. 135(3):880-887. 